Gláucia Virgína de Quetroz Lins Guerra

Alex Sandro Rolland de SOUza ${ }^{2}$

BRUNA FARIA DA COSTA ${ }^{3}$

Flávia Renata Quelroz do Nascimento ${ }^{3}$

Mariana de AndRade Amaral ${ }^{3}$

Ana Caroline Paz Serafim ${ }^{4}$

Artigo Original

Palavras-chave

Urinálise

Infecções urinárias/diagnóstico

Bacteriúria

Gravidez de alto risco

Keywords

Urinalysis

Urinary tract infections/diagnosis

Bacteriuria

Pregnancy, high-risk

\section{Exame simples de urina no diagnóstico de infecção urinária em gestantes de alto risco}

\author{
Urine test to diagnose urinary tract infection in highh-risk \\ pregant women
}

\section{Resumo}

OBJETIVO: Identificar a acurácia do exame simples de urina para diagnóstico de infecção urinária em gestantes de alto risco. MÉTODOS: Realizou-se um estudo transversal, prospectivo, em 164 gestantes admitidas na enfermaria de alto risco do Instituto de Medicina Integral Prof. Fernando Figueira (IMIP), no período de janeiro a junho de 2011 . Foram excluídas as pacientes em uso de antibiótico nos últimos dez dias. Todas as pacientes foram submetidas aos exames de urina simples e urocultura no início de sua admissão. A concordância entre os resultados dos exames foi avaliada pelo índice Kappa (K), sendo, ainda, determinados a acurácia, sensibilidade, especificidade e valor preditivo positivo (VPP) e negativo (VPN). RESULTADOS: Utilizando como critério de diagnóstico apenas a presença de piócitos no exame simples de urina para sugerir bacteriúria, observou-se uma fraca concordância quando comparado à urocultura $(K=0,16)$. A acurácia foi de $61 \%$, com sensibilidade de $62,5 \%$ e especificidade de $60,6 \%$. O VPP foi de $27,78 \%$ e o VPN, de 87\%. CONCLUSÃO: Na presença de alteração do exame simples de urina não necessariamente está em curso uma infecção urinária, sendo necessária a realização da urocultura. Porém, quando o exame simples de urina for normal, a urocultura pode ser dispensada.

\section{Abstract}

PURPOSE: To identify the accuracy of urinalysis in the diagnosis of urinary tract infection in pregnant women at high risk. METHODS: a prospective, cross-sectional study was conducted on 164 pregnant women admitted to the high-risk the ward of the Instituto de Medicina Integral Prof. Fernando Figueira (IMIP) during the period from January to June 2011. Patients who had been taking antibiotics in the last ten days were excluded. All patients were subjected to simple urine tests and urine culture at the beginning of their admission. The agreement between the results of the examinations was evaluated by Kappa indices (K), and accuracy, sensitivity, specificity, and positive (PPV) and negative (NPV) predictive values were also determined. RESULTS: When only the presence of pus cells in urinalysis was used as a diagnostic criterion suggesting bacteriuria, there was a poor agreement when compared to uroculture $(K=0.16)$. Accuracy was $61 \%$, sensitivity $62.5 \%$, and specificity $60.6 \%$. PPV was $27.78 \%$ and NPV was $87 \%$. CONCLUSION: The presence of alteration of urinalysis does not necessarily indicate an ongoing urinary tract infection, with urine culture being necessary. However, when urinalysis data are normal, uroculture may be avoided.
Correspondêncio

Alex Sandro Rolland de Souzo Rua dos Coelhos, 300 - Boa Visto CEP: $50070-550$ Recife (PE), Brasil

Recebido $03 / 08 / 2012$

Aceito com modificacões
Centro de Atenção à Mulher do Instituto de Medicina Integral Prof. Fernando Figueira - IMIP - Recife (PE), Brasil.

'Setor de Gestação de Alto Risco do Centro de Atenção à Mulher, Instituto de Medicina Integral Prof. Fernando Figueira - IMIP - Recife (PE), Brasil.

${ }^{2}$ Setor de Medicina Fetal do Centro de Atenção à Mulher, Instituto de Medicina Integral Prof. Fernando Figueira - IMIP - Recife (PE), Brasil. ${ }^{3}$ Curso de Medicina, Faculdade Pernambucana de Saúde - FPS - Recife (PE), Brasil.

4Programa de Residência Médica em Ginecologia e Obstetrícia, Instituto de Medicina Integral Prof. Fernando Figueira - IMIP - Recife (PE), Brasil.

Fonte de financiamento: Bolsistas do Programa Institucional de Iniciação Científica (PIBIC) do CNPq e IMIP.

Conflito de interesses: não há. 
Introdução

A infecção urinária caracteriza-se pela presença de agentes infecciosos e invasão dos tecidos urinários, sendo classificada segundo sua localização em infecção urinária baixa e alta. A bacteriúria assintomática é definida como o isolamento de bactérias na urina em quantidade maior ou igual a $10^{5}$ unidades formadoras de colônias por mililitros (UFC/mL), sem sinais ou sintomas locais ou sistêmicos de infecçãa ${ }^{1-3}$. Entre mulheres, gestantes ou não, sua prevalência é de 2 a $10 \%$, e encontra-se associada ao nível socioeconômico, história de infecção urinária recorrente, diabetes e anormalidades anatômicas do sistema urinário ${ }^{1}$.

Durante a gravidez, há uma série de alterações fisiológicas no organismo materno que leva a maior predisposição à infecção urinária e suas complicações. A estase urinária secundária, a compressão do ureter pelo útero gravídico e o relaxamento da musculatura por ação da progesterona favorecem o surgimento de bacteriúria, sendo um fator de risco importante para o desenvolvimento de pielonefrite e suas complicações durante a gravidez. Nessas mulheres, quando a bacteriúria, assintomática ou sintomática, não é tratada ou inadequadamente tratada, evolui para pielonefrite em $30 \%$ dos $\operatorname{casos}^{1,4,5}$. Estudos sugerem, ainda, que a bacteriúria, assintomática ou sintomática, encontra-se fortemente associada a complicações maternas e fetais, como a rotura prematura de membranas, trabalho de parto prematuro, corioamnionite, baixo peso ao nascer, febre materna e infecção neonatal ${ }^{4,5}$.

O exame mais utilizado para o diagnóstico de bacteriúria e infecção urinária é o exame simples de urina, também conhecido como sumário de urina e urinálise $e^{2,6}$. Este analisa a urina quanto à cor, densidade, aspecto, presença de leucócitos, bactérias, sangue, glicose, urobilinogênio, bilirrubina, nitrito e sedimentos urinários. Para esse diagnóstico provável, a literatura sugere que a presença de leucócitos, hemácias e nitrito são bons indicativos de bacteriúria ou infecção urinária. Entretanto, esses elementos são apenas sinais indiretos de inflamação, não sendo precisos para o diagnóstico definitivo de bacteriúria significativa. Para a confirmação de infecção urinária exige-se a cultura de urina, na qual o patógeno em crescimento é isolado e quantificado ${ }^{6}$.

Apesar de a literatura sugerir que o exame simples de urina apresenta um alto valor preditivo negativo, ou seja, quando ausentes sinais sugestivos de infecção urinária, aproximadamente $90 \%$ das mulheres encontram-se sem bacteriúria, as sociedades médicas internacionais ainda recomendam a realização da cultura de urina de rotina no rastreamento pré-natal da infecção urinária ${ }^{2,6-8}$.

Porém, diferentemente do exame simples de urina, que é de baixo custo, fácil acesso e realização, a urocultura não é disponibilizada de rotina na grande maioria dos serviços de pré-natal ${ }^{2}$. Assim, é importante haver estudos sobre a validação diagnóstica do exame simples de urina comparada à urocultura para reforçar o uso do melhor teste diagnóstico na rotina de pré-natal, visto que a bacteriúria, mesmo assintomática, quando não tratada de forma adequada poderá trazer grandes prejuízos para o binômio mãe e feto.

\section{Métodos}

Realizou-se um estudo analítico, prospectivo, do tipo transversal, envolvendo 164 gestantes admitidas na enfermaria de Gestação de Alto Risco do Instituto de Medicina Integral Prof. Fernando Figueira (IMIP), localizado em Recife, Pernambuco, Brasil, no período de janeiro a junho de 2011.

Foram incluídas as gestantes internadas na enfermaria de gestação de alto risco por motivos diversos, independentemente de apresentarem queixas urinárias. As pacientes em uso de antibiótico e/ou que o utilizaram nos últimos dez dias foram excluídas.

Todas as gestantes foram abordadas e esclarecidas quanto aos objetivos da pesquisa, sendo incluídas apenas após concordarem em participar e assinar o termo de consentimento livre e esclarecido. O estudo teve início apenas após aprovação pelo Comitê de Ética em Pesquisa em seres humanos do IMIP (CEP n ${ }^{\circ}$. 1.889-20, de outubro de 2010), sendo conduzida de acordo com a Declaração de Helsinque revisada em 2008.

O cálculo do tamanho amostral foi realizado utilizando o software Statcalc do Epi-info versão 3.5.1, prevendo-se uma frequência de bacteriúria assintomática de $10 \%^{2}$, com precisão de $30 \%$ e intervalo de confiança de $80 \%$ Encontrou-se um número de 164 participantes; prevendo-se eventuais perdas, optou-se por incluir no estudo 180 gestantes.

Assim, durante o estudo, foram candidatas à participação 211 gestantes, sendo 31 excluídas por uso de antibióticos no momento da admissão ou até 10 dias antes, e 16 foram perdidas por ausência de resultado de um dos exames após inclusão no estudo. Portanto, foram avaliadas 164 gestantes com idade entre 14 e 46 anos $(27,9 \pm 7,5$ anos) e idade gestacional variando entre 12 a 39 semanas (30,0 $\pm 5,9$ semanas) (Tabela 1 ).

A mediana da paridade foi um, e a de número de abortos anteriores foi zero, com $41,5 \%$ de mulheres nulíparas. A maioria das gestantes era proveniente do Recife e região metropolitana $(53,7 \%)$, sendo as demais do interior do Estado de Pernambuco e de outros Estados, 40,9 e $5,5 \%$, respectivamente. Em relação ao estado civil, $73,7 \%$ tinham companheiro, $22,6 \%$ eram solteiras e 3,7\% divorciadas. Os principais motivos da internação 
foram síndrome hipertensiva, diabetes e trabalho de parto prematuro (Tabela 1).

Para todas as participantes foram solicitados exames de urina simples, urocultura e aplicado um instrumento de coleta de dados no momento da admissão com as seguintes variáveis: idade, estado civil, procedência, paridade, idade gestacional, motivo da internação, presença de sinais e sintomas atuais de infecção urinária e resultado dos exames realizados.

O exame simples da urina foi realizado utilizando o aparelho Uriscan Pró II (Coreia) do laboratório de urinálise do IMIP e a análise foi automatizada. Avaliou-se a presença de nitrito, piócitos, hemácias (cinco ou mais hemácias por campo) e células epiteliais. Para o diagnóstico de bacteriúria/infecção urinária considerou-se como critério a presença de mais que quatro piócitos por campo e/ou nitrito positivo ${ }^{3}$.

A cultura de urina para isolamento e quantificação de microrganismos foi realizada no laboratório central de microbiologia do IMIP, conforme rotina estabelecida, segundo técnica de disco-difusão ${ }^{7}$. Considerou-se bacteriúria/infecção urinária quando a urocultura evidenciou mais que $10^{5}$ bactérias por colônia ${ }^{3,7}$.

A coleta da urina pela paciente foi realizada após instruções dadas pelos profissionais de saúde da enfermaria (técnicos de enfermagem ou enfermeiros). Colheu-se para o exame o jato intermediário da primeira urina do dia, após a realização de assepsia. $\mathrm{O}$ exame simples de urina e a urocultura foram realizados por profissionais do laboratório da instituição sem o conhecimento prévio do estudo ou da realização de ambos os exames.

A sintomatologia referida pela paciente foi definida como a presença de qualquer um dos sinais e sintomas, como disúria (dor ou ardor durante a micção), alteração do odor da urina, polaciúria (aumento na frequência urinária), hematúria (presença de sangue), febre (temperatura axilar maior que $37,5^{\circ} \mathrm{C}$ ) e dor lombar.

A analise estatística foi feita pelo programa Epi-Info versão 3.5.3 e Open-Epi versão 2.2. Para descrever as características da amostra foram utilizadas medidas de tendência central, dispersão e distribuição de frequências. Para validar o diagnóstico de bacteriúria/infecção urinária pelo exame simples de urina, foi determinada a acurácia (A), sensibilidade (S), especificidade (E), valor preditivo positivo (VPP) e negativo (VPN), razão de verossimilhança positiva e negativa e seus respectivos intervalos de confiança a 95\% (IC95\%).

Utilizou-se ainda o índice de Kappa (K) para se determinar a concordância entre os exames simples de urina e a urocultura, sendo considerada concordância ruim quando igual a 0,00 ; fraca, de 0,01 a 0,20 ; sofrível de 0,21 a 0,40 ; regular de 0,41 a 0,60 ; boa de 0,61 a 0,80 ; ótima de 0,81 a 0,99 ; e perfeita quando igual a $1,00^{8,9}$.

\section{Resultados}

Quando se considerou o critério diagnóstico de bacteriúria/infecção urinária apenas pela presença de mais que quatro piócitos por campo, o exame de urina simples foi considerado sugestivo de infecção em 72 mulheres $(43,9 \%)$, das quais $63(87,5 \%)$ eram assintomáticas. Porém, considerando a presença de piócitos e nitritros a bacteriúria/infecção urinária foi sugestiva em apenas seis gestantes $(3,6 \%)$, sendo três $(50,0 \%)$ assintomáticas. Destaca-se que todas as gestantes com nitrito positivo apresentavam quatro ou mais piócitos por campo.

$\mathrm{Na}$ urocultura, o exame foi positivo em 32 participantes $(19,5 \%)$ e apenas $7(21,9 \%)$ eram sintomáticas. O agente etiológico de maior frequência foi Escherichia coli, responsável por $28 \%$ das infecções, seguido de Klebsiella pneumoniae, Staphylococcus coagulase negativa e Streptococcus beta hemolítico, com 18,7, 18,7 e 15,6\%, respectivamente.

A presença de um ou mais sintomas foi relatado por 22 gestantes $(13,4 \%)$. Os sintomas mais frequentes foram disúria $(n=11 ; 6,7 \%)$, polaciúria $(n=9 ; 5,5 \%)$, hematúria

Tabela 1. Características das gestantes de alto risco no Instituto de Medicina Integral Prof. Fernando Figueira

\begin{tabular}{|c|c|}
\hline Características (n=164) & \\
\hline \multicolumn{2}{|l|}{ Idade (anos) } \\
\hline Média $\pm D P$ & $27,9 \pm 7,5$ \\
\hline Variação & $14-46$ \\
\hline \multicolumn{2}{|l|}{ Idade gestacional (semanas) } \\
\hline Média $\pm D P$ & $30,0 \pm 5,9$ \\
\hline Variação & $12-39$ \\
\hline \multicolumn{2}{|l|}{ Paridade } \\
\hline Mediana & 1 \\
\hline Variação & $0-11$ \\
\hline Nulíparas n (\%) & $68(41,5)$ \\
\hline \multicolumn{2}{|l|}{ Abortos } \\
\hline Mediana & 0 \\
\hline Variação & $0-3$ \\
\hline \multicolumn{2}{|l|}{ Procedência n (\%) } \\
\hline Recife e região metropolitana & $88(53,7)$ \\
\hline Interior de Pernambuco & $67(40,9)$ \\
\hline Outros estados & $9(5,5)$ \\
\hline \multicolumn{2}{|l|}{ Estado civil n (\%) } \\
\hline Com companheiro & $121(73,7)$ \\
\hline Solteira & $37(22,6)$ \\
\hline Divorciada & $6(3,7)$ \\
\hline \multicolumn{2}{|l|}{ Indicações de internamento n (\%)* } \\
\hline Síndrome hipertensiva & $60(36,6)$ \\
\hline Diabetes & $52(24,4)$ \\
\hline Trabalho de parto prematuro & $32(19,5)$ \\
\hline Rotura prematura das membranas & $11(6,7)$ \\
\hline Outros & $70(42,7)$ \\
\hline
\end{tabular}


Tabela 2. Validação diagnóstica do exame de urina simples com a urocultura em gestantes de alto risco do Instituto de Medicina Integral Prof. Fernando Figueira

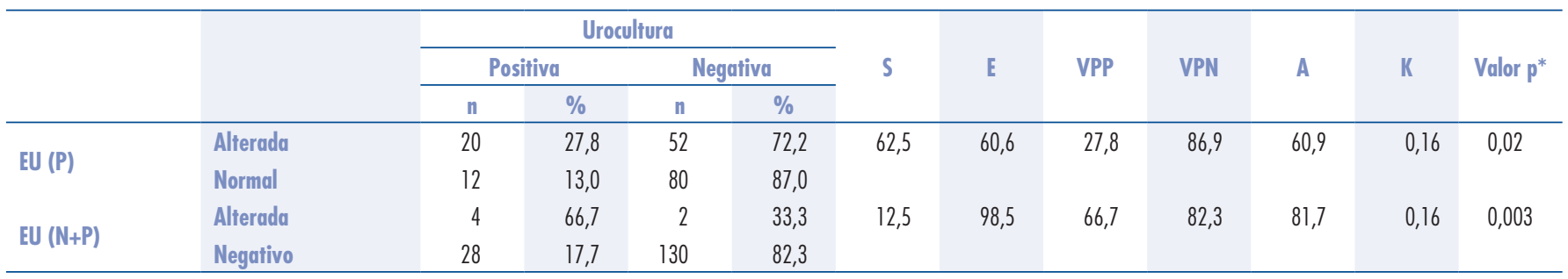

*Teste do $\chi^{2}$. EU: exame de urina; P: piócitos presentes; N+P: nitritos e piócitos presentes; S: sensibilidade; E: especificidade; VPP: valor preditivo positivo; VPN: valor preditivo negativo; A: acurácia; K: índice Kappa.

$(\mathrm{n}=8 ; 4,9 \%)$, dor lombar $(\mathrm{n}=7 ; 4,3 \%)$, febre $(\mathrm{n}=5 ; 3,0 \%)$ e odor forte $(\mathrm{n}=2 ; 1,2 \%)$.

Quando o exame simples de urina foi sugestivo de infecção, utilizando como critério apenas a presença de piócitos, em $27,8 \%$ das pacientes a urocultura foi positiva. Quando o exame simples de urina foi normal, em 87,0\% das vezes a urocultura foi negativa. Assim, observou-se um índice de Kappa de 0,16 (IC95\% 0,03-0,3), com acurácia de 61,0\% (IC95\% 53,3-68,1), sensibilidade de $62,5 \%$ (IC95\% 45,2-77,1), especificidade de 60,6\% (IC95\% 52,1-68,5), valor preditivo positivo $27,8 \%$ (IC95\% 18,8-39,0), valor preditivo negativo de 87,0\% (IC95\% 78,6-92,4), razão de verossimilhança positiva de 1,6 (IC95\% 1,4-1,7) e razão de verossimilhança negativa de 0,6 (IC95\% 0,5-0,7) (Tabela 2).

Quando se analisou a presença de piócitos associada à presença de nitrito o índice de Kappa foi de 0,16 (IC95\% 0,05-0,3), com acurácia de 81,7\% (IC95\% 53,3-68,1), sensibilidade de $12,5 \%$ (IC95\% 5,0-28,1), especificidade de 98,5\% (IC95\% 94,6-99,6), valor preditivo positivo $66,8 \%$ (IC95\% 30,0-90,3), valor preditivo negativo de 82,3\% (IC95\% 75,6-87,4), razão de verossimilhança positiva de 8,2 (IC95\% 0,1-678,7) e razão de verossimilhança negativa de 0,9 (IC95\% 0,8-0,9) (Tabela 2).

\section{Discussão}

Em nossa pesquisa, analisamos a acurácia do exame de urina simples no diagnóstico de infecção urinária a partir de amostra representativa de gestantes atendidas em uma enfermaria de gestação de alto risco. As sociedades médicas americanas recomendam a realização de rotina da urocultura para o rastreio de bacteriúria assintomática durante a gestaçãa ${ }^{10,11}$. O Ministério da Saúde do Brasil indica que a urocultura seja reservada quando o exame simples de urina sugira infecção do trato urinário ou quando o médico julgar necessário ${ }^{12}$.

$\mathrm{O}$ American College of Obstetrics and Gynecology recomenda que a cultura de urina seja realizada na primeira consulta pré-natal e repetida no $3^{\circ}$ trimestre de gravidez ${ }^{10}$, enquanto, a recomendação do U.S. Preventive Services Task
Force é que a urocultura seja realizada entre a $12^{\mathrm{a}}$ e $16^{\mathrm{a}}$ semanas de gestação ${ }^{11}$.

As dúvidas em relação ao exame de rastreamento de infecção urinária ou bacteriúria assintomática ${ }^{10-12}$ e a maior frequência dessas entidades clínicas em gestações de alto risco motivaram a realização dessa pesquisa nesse grupo de mulheres ${ }^{13,14}$. De forma semelhante a outros estudos nos quais se incluíram gestantes de alto risco ${ }^{15,16}$, as mulheres investigadas em nossa pesquisa eram jovens, apresentando média de idade de 27,9 anos, 41,4\% delas eram primíparas e $27,4 \%$ declaravam antecedente de pelo menos um aborto. A maioria provinha do Recife e região metropolitana e viviam com companheiro.

Síndromes hipertensivas, diabetes, trabalho de parto prematuro e rotura prematura das membranas foram os principais diagnósticos. Tais frequências estão de acordo com estudos realizados em enfermarias de gestação de alto risco $^{15}$, da mesma forma que o principal diagnóstico na internação foi a hipertensão durante a gravidez, provavelmente porque as síndromes hipertensivas são bastante frequentes em nosso meio ${ }^{15,16}$.

Diabetes, trabalho de parto prematuro e rotura prematura das membranas, além de serem importantes causas de internação pré-natal, também estão associadas à bacteriúria/infecção urinária sintomática ou não ${ }^{1}$. Estudos sugerem uma associação significativa de causa e efeito da bacteríuria/infecção e o parto prematuro e rotura prematura das membranas ${ }^{17,18}$. Por seu lado, o diabetes está associado à instalação de processos infecciosos, particularmente a bacteriúria/infecção urinária ${ }^{19}$.

Em nosso estudo a cultura de urina foi positiva em $19,5 \%$ das pacientes. Resultados esses semelhantes à maioria dos estudos ${ }^{2,14,20}$. Da mesma forma, o agente etiológico mais frequente foi a Escherichia coli, quando comparado a outros estudos ${ }^{2,21,22}$. Porém a frequência de Escherichia coli foi aproximadamente $28 \%$, menor que a encontrada em outros ${ }^{2,22}$. Pode-se atribuir a esse fato a diferença das populações incluídas nos estudos:.este em ambiente hospitalar, e os outros em ambiente ambulatorial. Além disso, vários estudos vêm descrevendo a mudança do perfil etiológico dessa infecção. Estudo realizado no 
próprio IMIP descreveu um aumento na frequência de Klebsiela sp. em mulheres internadas não gestantes ${ }^{7}$. Tal fato também pode estar ocorrendo entre as gestantes.

Em relação aos sinais e sintomas relativos à infecção urinária, referidos pelas gestantes, constatamos que apenas $21,9 \%$ das gestantes com urocultura positiva apresentaram algum tipo de sintomatologia. Porém, analisar sinais e sintomas de infecção do trato urinário (ITU) durante a gestação é por vezes difícil para o profissional de saúde assistente, já que muitos deles são habitualmente referidos pelas gestantes, não caracterizando ITU ${ }^{20}$. Um estudo observou que, das 232 mulheres incluídas, 46,5\% relataram sintomas urinários, porém apenas $10(4,3 \%)$ apresentaram crescimento bacteriano na cultura de urina. Assim, na maioria das mulheres os sintomas foram atribuídos a mudanças fisiológicas induzidas no sistema urinário durante a gravidez ${ }^{21}$. Em relação à maior frequência de infecção urinária sintomática em nosso estudo, podemos justificar pela amostra de gestantes de alto risco e devido à metodologia dos estudos ser diferente.

De forma semelhante a outros autores, os sintomas mais referidos pelas pacientes foram a disúria e polaciúria ${ }^{21}$. Porém, a completa ausência de sintomas não descarta a presença de bacteriúria e, consequentemente, os riscos dela decorrentes ${ }^{21,23}$. Assim, destacamos a importância da realização do diagnóstico de bacteriúria/infecção urinária independente da presença ou ausência de sintomas durante a gravidez, desencorajando o início do tratamento apenas baseado na sintomatologia clínica.

A bacteriúria assintomática pode evoluir para pielonefrite em 25 a $40 \%$ dos casos, se a paciente não for trata$\mathrm{da}^{13,23}$. Das 164 gestantes analisadas no presente estudo, a frequência de bacteriúria assintomática foi de 15,2\%, estando acima da referida na literatura ${ }^{14,21,23}$. Isso pode ser explicado pela população do nosso estudo - mulheres internadas no setor de gestação de alto risco.
Sabendo-se do risco aumentado para o desenvolvimento de ITU na gestação, da possibilidade de ocorrência de bacteriúria assintomática e das possíveis complicações maternas e perinatais, alguns pesquisadores apontam como inquestionável a necessidade de realização de urocultura, rotineiramente, no início da gestaçãa ${ }^{10,11}$. Entretanto, a urocultura exige laboratórios e profissionais especializados, sendo sua realização difícil em alguns dos serviços de pré-natal do Brasil, particularmente nas regiões menos desenvolvidas e nas Unidades Básicas de Saúde.

Um estudo realizado na Índia afirma que a urocultura pode ser solicitada apenas quando o exame simples de urina estiver alterado ${ }^{2}$, que está de acordo com o resultado encontrado em nossa população. Observamos que, quando o exame simples de urina foi normal (utilizando apenas o critério de piócitos), apenas em $13 \%$ a cultura de urina foi discordante - isto é, positiva — resultando em um valor preditivo negativo para o exame simples de urina de $86,9 \%$. Porém, é importante destacar que, quando acrescentamos à presença de nitritos como critério diagnóstico, a especificidade do exame simples de urina aumenta de 62,5 para $98,5 \%$, mantendo o valor preditivo negativo semelhante. Vários estudos encontraram resultados semelhantes ao nosso: alto valor preditivo negativo, uma boa especificidade, porém baixa sensibilidade, e baixo valor preditivo positivo $2,14,20,24,25$.

Assim, após analisar o valor preditivo positivo e o valor preditivo negativo do exame simples de urina, concluiu-se que na presença de alteração do exame não necessariamente está em curso uma infecção urinária, sendo necessária a realização da urocultura. Quando o exame simples de urina for normal, o resultado dessa pesquisa indicou que em $87 \%$ das vezes a urocultura pode ser dispensada. Assim, nossos dados sugerem que em locais onde há impossibilidade de realização da urocultura, o exame simples de urina pode ser utilizado como rastreio de infecção urinária.

\section{Referências}

1. Smaill FM, Vazquez JC. Antibiotics for asymptomatic bacteriuria in pregnancy. Cochrane Database Syst Rev. 2011 ; (1):CD000490.

2. Jayalakshmi J, Jayaram VS. Evaluation of various screening tests to detect asymptomatic bacteriuria in pregnant women. Indian J Pathol Microbiol. 2008;51(3):379-81.

3. Carvalhal GF, Rocha LCA, Monti PR. Urocultura comum de urina: considerações sobre sua coleta e interpretação. Rev AMGRIS. 2006;50(1):59-62.

4. Villar J, Widmer M, Lydon-Rochelle M, Gülmezoglu AM, Roganti A. Duration of treatment for asymptomatic bacteriuria during pregnancy. Cochrane Database Syst Rev. 2011 ; (1):CD000491.
5. Vazquez JC, Abalos E. Treatments for symptomatic urinary tract infections during pregnancy. Cochrane Database Syst Rev. $2011 ;(1)$ :CD002256.

6 Feitosa DCA, Silva MG, Parada CMGL. Acurácia do exame de urina simples para diagnóstico de infecções do trato urinário em gestantes de baixo risco. Rev Latino-Am Enfermagem. 2009; 17(4):507-13.

7. Hinrichsen SC, Amorim MMR, Souza ASR, Costa A, Hinrichsen MGML, Hinrichsen SL. Perfil dos microorganismos isolados no trato urinário após sondagem vesical em cirurgia ginecológica. Rev Bras Saúde Matern Infant. 2009;9(1):77-84. 
8. Organização Pan-Americana de Saúde. Fundação Nacional de Saúde. Centro Nacional de Epidemiologia. Métodos de investigação epidemiológica em doenças transmissíveis: manual do instrutor. Brasília (DF): FUNASA; 1997.

9. Viera A, Garrett JM. Understanding interobserver agreement: the kappa statistic. Fam Med. 2005;37(5):360-3.

10. American College of Obstetricians and Gynecologists. ACOG educational bulletin. Antimicrobial therapy for obstetric patients. Number 245, March 1998 (replaces no. 117, June 1988). Int J Gynaecol Obstet. 1998;61(3):299-308.

11. United States Preventive Services Task Force. Guide to clinical preventive services: periodic updates. 3rd ed. Rockville: Agency for Healthcare Quality and Research; 2004.

12. Schirmer J. Assistência pré-natal: manual técnico. 3a ed. Brasília (DF): Secretaria de Políticas de Saúde/Ministério da Saúde; 2000.

13. Paula LG, Krahe C, Carvalho RL. Infecção urinária e gestação. Femina. 2005;33(3):209-13.

14. Bandyopadhyay S, Thakur JS, Ray P, Kumar R. High prevalence of bacteriuria in pregnancy and its screening methods in north India. J Indian Med Assoc. 2005; 103(5):259-62, 266.

15. Souza ASR, Scavuzzi A, Rodrigues DC, Oliveira RD, Feitosa FEL, Amorim MMR. Titrated oral solution of misoprostol for labour induction: a pilot study. Solução oral escalonada de misoprostol para indução do parto: estudo piloto. Rev Bras Ginecol Obstet. 2010;32(5):208-13.

16. Amorim $M M R$, Katz L, Ávila MB, Araújo DE, Valença $M$, Albuquerque CJM, et al. Perfil das admissões em uma unidade de terapia intensiva obstétrica de uma maternidade brasileira. Rev Bras Saúde Matern Infant. 2006;6 Supl 1: S55-S62.
17. Hernández y Ballinas A, López Farán JA, Gámez Guevara C. Comparación de resultados maternos y perinatales en el tratamiento conservador de la rotura prematura de membranas pretérmino entre el uso de eritromicina y clindamicina. Ginecol Obstet Mex. $2011 ; 79(7): 403-10$

18. O'Neill MS, Hertz-Picciotto I, Pastore LM, Weatherley BD. Have studies of urinary tract infection and preterm delivery used the most appropriate methods? Paediatr Perinat Epidemiol. 2003; 17(3):226-33.

19. Fünfstück R, Nicolle LE, Hanefeld M, Naber KG. Urinary tract infection in patients with diabetes mellitus. Clin Nephrol. 2012;77(1): 40-8.

20. Teppa R, Roberts JM. The uriscreen test to detect significant asymptomatic bacteriuria during pregnancy. J Soc Gynecol Investig. $2005 ; 12(1): 50-3$.

21. Haider $G$, Zehra N, Munir AA, Haider A. Risk factors of urinary tract infection in pregnancy. J Pak Med Assoc. 2010;60(3):213-6.

22. D'Souza Z, D'Souza D. Urinary tract infection during pregnancy - dipstick urinalysis vs. culture and sensitivity. J Obstet Gynaecol. 2004;24(1):22-4.

23. Mittal $P$, Wing DA. Urinary tract infections in pregnancy. Clin Perinatol. 2005;32(3):749-64.

24. Bachman JW, Heise RH, Naessens JM, Timmerman MG. A study of various tests to detect asymptomatic urinary tract infections in an obstetric population. JAMA. 1993;270(16):1971-4.

25. Holland DJ, Bliss KJ, Allen CD, Gilbert GL. A comparison of chemical dipsticks read visually or by photometry in the routine screening of urine specimens in the clinical microbiology laboratory. Pathology. $1995 ; 27(1): 91-6$. 